\title{
AmBird: Mediating Intimacy for Long Distance Relationships through an Ambient Awareness System
}

\author{
Sine Jespersen, Rasmus Stounbjerg, Nervo Verdezoto \\ Department of Computer Science, Aarhus University \\ Aabogade 34 D, 8200 Aarhus N, Denmark \\ \{sine90, rbks, nervo\}@cs.au.dk
}

\begin{abstract}
This paper introduces the AmBird concept that explores how to provide alternatives for mediating intimacy for people that are living apart. The initial design and implementation of the AmBird concept is described as well as a preliminary concept validation. Based on the lessons learned, we highlight the opportunities of AmBird to support multiple intimate acts and our future work.
\end{abstract}

\section{Author Keywords}

Ambient Awareness; Intimacy; Communication

\section{ACM Classification Keywords}

H.5.m. Information interfaces and presentation (e.g., HCI): Miscellaneous.

\section{INTRODUCTION}

Nowadays, there are several tools such as Skype, Facebook, and text messages that provide people with various channels of communication. Although these technologies support people's communication needs in a more synchronous and explicit way, they require dedicated user attention and might not be sufficient to support the communication within intimate relationships [8]. Couples, friends or relatives living apart rarely share the same physical space $[1,8]$, and therefore they often do not get the opportunity to engage, and perform intimate acts [8]. Rather than using screen-based interaction [4], ambient information systems (AIS) [7] and physical interaction [9] can provide people with a sense of intimacy while supporting social and peripheral awareness (e.g., presence, activity, etc.) through the augmentation of everyday artifacts or dynamic changes in physical spaces $[1,6,9]$, that better fit everyday settings [9]. However, most of these systems have been designed to support minimal communication (a discrete change in the physical artifact [9]) or used a screen-based display to send simple messages [1]. As such, there is a critical need to support long distance relationships with more asymmetric [1], simple, and implicit alternatives for sending intimate messages with a higher bandwidth (more content) [9] through physical

Copyright $(\mathrm{C} 2015$ is held by the author(s). Publication rights licensed to Aarhus University and ACM

5th Decennial Aarhus Conference on Critical Alternatives

August 17-21, 2015, Aarhus Denmark

DOI: http://dx.doi.org/10.7146/aahcc.v1i1.21321

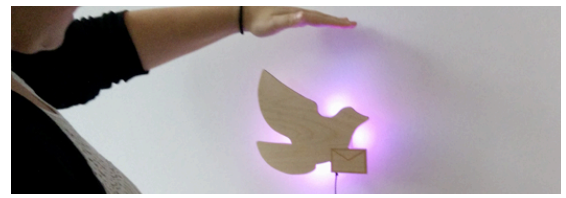

Figure 1. AmBird placed over a bed

interaction. Rather than using a dedicated display [1], this paper explores how the physicality of an AIS can provide alternatives to perform multiple intimate acts, while sending color messages to promote active reinterpretation [4].

THE AMBIRD: CONCEPT AND ITERATIVE DESIGN

The AmBird concept is designed to support multiple intimate acts between couples, friends or relatives living apart to increase their interpersonal awareness [9]. Initially, we identified the main requirements as having to be aesthetically pleasing for the home and be able to send intimate messages in an unobtrusive manner (ambient, tangible, and implicit [7]). It should support secrecy, reciprocity, and expressiveness [8] in a more pleasurable way [2]. Our iterative design process moved back and forth from brainstorming sessions using low fidelity prototypes to a more physical computing design process to support discussions and get early feedback [3]. AmBird is an augmented, wall-mounted, plywood bird that could be placed anywhere at home, but preferably in the bedroom (see Figure 1). AmBird is inspired by the associated communication properties of carrier pigeons. To support reciprocity [8], AmBird is wirelessly connected in pairs and used to send color messages as intimate acts back and forth between two people. Although we have defined a fixed set of colors, the undefined semantic can enable people to create their own interpretations [4] regarding the feelings associated with them to provide a playful experience [2]. To support expressiveness [2], AmBird changes the color according to the user's hand position and sends a color by whistling to it. Regarding secrecy, AmBird balances the distinction between public/private spaces so that a couple can engage in intimate acts in public without being noticed [8] e.g., in case of visitors, they might perceive the color without understanding its meaning.

\section{SYSTEM DESCRIPTION}

AmBird consists of two physical computing modules - one per bird (laser cut). Each module has an Arduino Yún for managing input and output sensors. An infrared sensor is used to change the colors according to the position and 
distance of the hand on top of each bird. The primary outputs are RGB LEDs, which illuminate the back of each bird. It is possible to choose from six distinct colors by moving the hand up and down above the bird. When a color is selected, the user holds his/her hand above the bird and whistles into a microphone placed in the bird's beak to send a color message. After the color is sent, the RGB LEDs on the back flash three times in the selected color to confirm that the message has been sent. When the associated AmBird (the pair) has received the message, it lights up in the received color and stays lit in the background, until the user waves his/her hand above to turn it off. The contacted person can choose not to turn its light off if the user wishes to have the received message visible for a longer time. All electronic components are placed in a box on the back of each bird and powered by a USB cable.

\section{CONCEPT VALIDATION}

We have performed a preliminary concept validation with a couple (aged 25 and 22) who has been in a relationship for 18 months and has recently moved in together. Although our couple is not living apart, we used AmBird as a trigger [5] to receive early feedback on the concept and facilitate discussions. After introducing AmBird, we performed a focus group and asked them to perform a semantic task ${ }^{1}$ based on their communication habits. We did not require the couple to show us the result of the task as this would have been obtrusive regarding their privacy. After the task, they proceeded to evaluate AmBird in separate rooms, sending color messages by themselves. In total, the couple interacted with the two modules for ten minutes, and then shared their experiences with us.

Overall, the participants found the semantic task amusing, and they often glanced upon each other and laughed when checking their picked sentences. Furthermore, the couple found the color-coding very nuanced as pointed out by the female participant: "colors seem more real than reading a text." She also explained that she never pays much attention to her texts messages. Both participants found AmBird easy to use and agreed that having a device that did not demand immediate attention and a response, like a phone, would be beneficial to communicate intimate messages. We also found that six colors were sufficient to cover the messages that the couple wanted to communicate. Although both participants liked the AmBird's physical interaction, the male participant was concerned about the possibility of losing messages as the latest color overrides the previous one. Also he reported that changing between colors was a bit unsmooth. The female participant thought it would be nice to get a sound notification when receiving a message. About the overall concept, the female participant expressed "You can better feel something wholeheartedly with colors than with SMS", and the male stated, "When you get a text message, everything is spelled out for you, but you interpret the underlying tone yourself. When you get a color you think more about the feeling of the message."

\section{CONCLUSION AND FUTURE WORK}

Our participants were positive about the concept and they were able to interact and send colors with AmBird. Although our preliminary evaluation provides us with positive early feedback, future work should consider that not all couples would necessarily have the same experience or be able to create their own semantics, so future studies are needed in more distant settings. As Kaye et al. [4] demonstrate, one bit communication could provide a valid communication channel for couples, and our findings highlight that extending one bit communication using color as an alternative is possible without making the interaction too demanding. In the next iteration, AmBird will be tested for a longer period with people living apart to acquire a better understanding of its capabilities and user experience.

\section{REFERENCES}

1. Brereton, M., Soro, A., Vaisutis, K. and Roe, P. The Messaging Kettle: Prototyping Connection over a Distance between Adult Children and Older Parents. In Proc.CHI (2015), 713-716.

2. Costello, B. and Edmonds, E. A study in play, pleasure and interaction design. In Proc. DPPI (2007), 76-91.

3. Hallgrimssom, B. Prototyping and modelmaking for product design. Laurence King Publ., 2012.

4. Kaye, J. J., Levitt, M. K., Nevins, J., Golden, J. and Schmidt, V. Communicating intimacy one bit at a time. In Proc. CHI '05 Extended Abstracts (2005).

5. Mogensen, P. and Trigg, R. H. Using artifacts as triggers for participatory analysis. In Proc. PDC (1992), 55-62.

6. Park, J.-Y. and Nam, T.-J. Dynamic design elements for the peripheral interaction of ambient media. In Proc. CHI '08 Extended Abstracts (2008), 3717-3722.

7. Pousman, Z. and Stasko, J. A taxonomy of ambient information systems: four patterns of design. In Proc. AVI (2006), 67-74.

8. Vetere, F., Gibbs, M. R., Kjeldskov, J., Howard, S., Mueller, F. F., Pedell, S., Mecoles, K. and Bunyan, M. Mediating intimacy: designing technologies to support strong-tie relationships. In Proc.CHI (2005), 471-480.

9. Visser, T., Vastenburg, M. H. and Keyson, D. V. Designing to support social connectedness: The case of SnowGlobe. International Journal of Design, 5, 3 (2011), 129-142.

\footnotetext{
1 "Pick ten sentences that you would like to say to your partner throughout the day, write them on a piece of paper, and do not show each other. Then, assess which sentences are most important. Read each other's sentences, and try to reach a common list of sentences. If possible, assign these sentences distinct colors."
} 